\title{
INTERVAL GAUSSIAN ELIMINATION WITH PIVOT TIGHTENING*
}

\author{
JÜRGEN GARLOFF ${ }^{\dagger}$
}

\begin{abstract}
We present a method by which the breakdown of the interval Gaussian elimination caused by division of an interval containing zero can be avoided for some classes of matrices. These include the inverse nonnegative matrices, the totally nonnegative matrices, and the inverse $M$-matrices - all classes with identically signed inverses. The approach consists of a tightening of the interval pivot by determining the exact range of the pivot over the matrix interval.
\end{abstract}

Key words. interval Gaussian elimination, inverse nonnegative matrix, totally nonnegative matrix, inverse $M$-matrix

AMS subject classifications. 65G20,65G30, 15A48

DOI. $10.1137 / 080729621$

1. Introduction. Systems of linear interval equations arise when the entries of the coefficient matrix and the right-hand side of a system of linear equations are varying between given bounds; cf. [20, sect. 3.4]. The solution set of such a system

$$
[A] x=[b],
$$

where $[A]=[\underline{A}, \bar{A}]$ is a given $n$-by- $n$ matrix interval and $[b]=[\underline{b}, \bar{b}]$ is a given vector interval w.r.t. the usual entrywise partial order, is the set

$$
\Sigma([A],[b]):=\left\{x \in \mathbf{R}^{n} \mid A x=b, A \in[A], b \in[b]\right\} .
$$

We will assume here throughout that all $A \in[A]$ are nonsingular. We denote the hull of $\Sigma([A],[b])$, i.e., the smallest vector interval containing $\Sigma([A],[b])$, by $[A]^{H}[b]$. A method to enclose $[A]^{H}[b]$ is interval Gaussian elimination, which is obtained from the usual (termed ordinary henceforth) Gaussian elimination by replacing the real numbers by the related intervals and the real operations by the respective interval operations; see, e.g., [1, Chap. 15], [20, sect. 4.5]. However, interval Gaussian elimination may fail due to division by an interval pivot containing zero, even when ordinary Gaussian elimination works for all matrices $A \in[A]$. There are some classes of interval matrices for which interval Gaussian elimination cannot fail, e.g., the $H$-matrices; cf. [17].

If ordinary Gaussian elimination is applied without pivoting, the pivots can be represented as the quotient of two successive leading principal minors. This property is used in [18] to modify the interval Gaussian elimination by tightening the interval pivots, and it is shown that the breakdown of interval Gaussian elimination can be avoided in some cases. However, this tightening is obtained by bounding the range of the two leading principal minors independently and then forming the quotient of both enclosures (in addition, the resulting interval is intersected with the ordinary interval

${ }^{*}$ Received by the editors July 9, 2008; accepted for publication (in revised form) by A. Frommer October 29, 2008; published electronically February 20, 2009. This work was supported by grants from the state of Baden-Württemberg, Germany.

http://www.siam.org/journals/simax/30-4/72962.html

$\dagger$ Faculty of Computer Science, University of Applied Sciences/HTWG Konstanz, Postfach 100543, D-78405 Konstanz, Germany (garloff@htwg-konstanz.de). 
pivot). This often causes an overestimation of the range of the pivot. In this paper, we show that for some classes of matrices the exact range of the pivot can be given. As a consequence of the inclusion isotonicity of the interval arithmetic operations, this further tightening of the interval pivot has the additional advantage that the resulting enclosure of $[A]^{H}[b]$ is not larger than the one obtained by the method of [18] and it is often smaller. Moreover, the range of the pivots can be obtained by running a few ordinary Gaussian elimination procedures in parallel.

The organization of the paper is as follows: In the next section we introduce our notation and recall the interval Gaussian elimination. In section 3 we present pivot tightening. This is applied in section 4 to inverse nonnegative matrices, to totally nonnegative matrices, and to inverse $M$-matrices. In sections 5 and 6 we show that analogous results can be obtained for a related determinantal function and some algorithms for the solution of structured systems of linear interval equations. We conclude with some remarks in section 7 .

2. Notation and interval Gaussian elimination. By $\mathbf{R}^{n}, \mathbf{R}^{n \times n}, \mathbf{I R}, \mathbf{I R}^{n}$, and $\mathbf{I R}^{n \times n}$ we denote the set of real vectors with $n$ components, the set of real $n$-by- $n$ matrices, the set of the compact and nonempty intervals, the set of the intervals of real vectors with $n$ components, and the set of the intervals of real $n$-by- $n$ matrices, respectively. We also regard vector intervals as interval vectors and matrix intervals as interval matrices, i.e., as vectors and matrices over IR, respectively, and consequently represent them as $[b]=[\underline{b}, \bar{b}]=\left(\left[b_{i}\right]\right)_{i=1}^{n}=\left(\left[\underline{b}_{i}, \bar{b}_{i}\right]\right)_{i=1}^{n}$ and $[A]=[\underline{A}, \bar{A}]=\left(\left[a_{i j}\right]\right)_{i, j=1}^{n}=$ $\left(\left[\underline{a}_{i j}, \bar{a}_{i j}\right]\right)_{i, j=1}^{n}$. We identify a degenerate interval (vector, matrix) with the (only) real number (vector, matrix) it contains.

We equip IR, $\mathbf{I R}^{n}$, IR $\mathbf{I R}^{n \times n}$ with the usual real interval arithmetic; see, e.g., [1, Chap. 10], [20, Chap. 1]. We assume that the reader is familiar with the basic properties of this arithmetic. For a function $f: \mathbf{R}^{n \times n} \rightarrow \mathbf{R}$ we denote the range of $f$ over the matrix interval $[A]$ by $f([A])$, i.e.,

$$
f([A]):=\{f(A) \mid A \in[A]\} .
$$

The interval Gaussian elimination (without pivoting) reads as follows.

Given $[A] \in \mathbf{I R}^{n \times n},[b] \in \mathbf{I R}^{n}$, define $[A]^{(k)}=\left(\left[a_{i j}\right]^{(k)}\right) \in \mathbf{I R}^{n \times n},[b]^{(k)}=$ $\left(\left[b_{i}\right]^{(k)}\right) \in \mathbf{I R}^{n}, k=1, \ldots, n$, and $[x]^{G}=\left(\left[x_{i}\right]^{G}\right) \in \mathbf{I R}^{n}$ by

$$
\begin{gathered}
{[A]^{(1)}=[A], \quad[b]^{(1)}=[b],} \\
{\left[a_{i j}\right]^{(k+1)}= \begin{cases}{\left[a_{i j}\right]^{(k)},} & i=1, \ldots, k, j=1, \ldots, n, \\
{\left[a_{i j}\right]^{(k)}-\frac{\left[a_{i k}\right]^{(k)} \cdot\left[a_{k j}\right]^{(k)}}{\left[a_{k k}\right]^{(k)}},} & i=k+1, \ldots, n, j=k+1, \ldots, n, \\
0 & \text { otherwise, }\end{cases} } \\
{\left[b_{i}\right]^{(k+1)}= \begin{cases}{\left[b_{i}\right]^{(k)},} & i=1, \ldots, k, \\
{\left[b_{i}\right]^{(k)}-\frac{\left[a_{i k}\right]^{(k)}}{\left[a_{k k}\right]^{(k)}} \cdot\left[b_{k}\right]^{(k)},} & i=k+1, \ldots, n,\end{cases} } \\
{\left[x_{i}\right]^{G}=\left(\begin{array}{ll}
{\left[b_{i}\right]^{(n)}-\sum_{j=i+1}^{n}\left[a_{i j}\right]^{(n)}\left[x_{j}\right]^{G}}
\end{array}\right) /\left[a_{i i}\right]^{(n)}, \quad i=n, n-1, \ldots, 1,}
\end{gathered}
$$


where $\sum_{j=n+1}^{n} \cdots=0$. Note that no rows or columns are permuted. The algorithm is feasible if and only if $0 \notin\left[a_{k k}\right]^{(k)}, k=1, \ldots, n$.

For a survey on results on this algorithm the reader is referred to [17]. Since we are interested only in the feasibility of the algorithm we consider here only the formulae in (2.1) which provide the transformation of $[A]$ into triangular form.

We further adopt standard notation from matrix analysis. For any $n$-by- $n$ ma$\operatorname{trix} A$, we denote the submatrix lying in rows indexed from $\alpha$ and columns indexed from $\beta$ (both in increasing order) by $A[\alpha \mid \beta]$, where $\alpha, \beta \subseteq\{1,2, \ldots, n\}$. If $\alpha=\beta$, the principal submatrix $A[\alpha \mid \alpha]$ is abbreviated as $A[\alpha]$. In particular, when $\alpha=\{1, \ldots, k\}, k=1, \ldots, n$, we call $A[\alpha]$ a leading principal submatrix and put $A^{\prime}:=A[\{1,2, \ldots, n-1\}]$. We denote by $A_{i j}$ the submatrix of $A$ which is obtained by the deletion of row $i$ and column $j$ in $A$. We call $A$ a $P$-matrix if all its principal minors are positive, inverse nonnegative ${ }^{1}$ if it is nonsingular and its inverse is entrywise nonnegative, and an $M$-matrix if it is inverse nonnegative and all its off-diagonal entries are nonpositive. If $\pi$ is a certain property of a matrix, then we call a matrix interval $[A]=[\underline{A}, \bar{A}]$ a $\pi$ matrix interval if all $A \in[A]$ possess property $\pi$. For example, $[A]$ is an inverse $M$-matrix interval if all $A \in[A]$ are inverse $M$-matrices. Often it is of interest to know whether such a property of a matrix interval can be inferred from a certain subset of the vertex matrices $A=\left(a_{i j}\right)$ with $a_{i j} \in\left\{\underline{a}_{i j}, \bar{a}_{i j}\right\}$, for all $i, j$. Examples are given in section 4.

3. Interval pivot tightening. Interval Gaussian elimination breaks down when an interval pivot $\left[a_{k k}\right]^{(k)}$ (henceforth termed ordinary interval pivot) contains zero. This can occur even though all matrices $A \in[A]$ have nonvanishing leading principal minors [22]. For ordinary Gaussian elimination, the pivot $a_{k k}^{(k)}$ can be represented as the quotient of two succeeding leading principal minors (e.g., [5, p. 26]),

$$
a_{k k}^{(k)}=\frac{\operatorname{det} A[\{1,2, \ldots, k\}]}{\operatorname{det} A[\{1,2, \ldots, k-1\}]} .
$$

This property is used in [18] to tighten the ordinary interval pivot $\left[a_{k k}\right]^{(k)}$ : Let $D_{k}$ denote an enclosure for $\operatorname{det} A[\{1,2, \ldots, k\}]$ for all $A \in[A]$, where $D_{0}:=1$. If

$$
0 \notin D_{k}, \quad k=1,2, \ldots, n,
$$

then $\left[a_{k k}\right]^{(k)}$ in $(2.1)$ is replaced by

$$
\left[a_{k k}\right]^{(k)} \cap \frac{D_{k}}{D_{k-1}} .
$$

In general, this approach does not have any advantage over the ordinary interval pivot because finding an enclosure for the range of a determinant is as difficult as the original problem of solving a system of linear interval equations. However, for some classes of interval matrices presented in section 4 the exact range of principal minors can be given (only by using two specified vertex matrices) and (3.2) is satisfied. Then, when (3.3) is used as the interval pivot the breakdown of interval Gaussian elimination can be avoided. As a welcome side effect, the enclosure of the solution set may be tighter compared to the one which is obtained when the ordinary interval pivot is

\footnotetext{
${ }^{1}$ This is often also called inverse positive; since we use the terminology totally nonnegative instead of totally positive (cf. subsection 4.2), we consequently prefer inverse nonnegative.
} 
used; at least it will not be larger. This is a consequence of the inclusion isotonicity of the interval arithmetic operations.

However, by enclosing the ranges of the two principal minors in (3.1) independently some overestimation is introduced into the computation. In the following, we identify some classes of matrices for which the range of the pivot $a_{k k}^{(k)}$ for all $A \in[A]$ can be given exactly such that forming the intersection in (3.3) is not necessary. Again, as a side effect, this further tightening may result in a further shrinking of the enclosure of the solution set (1.1).

The classes of matrices we will discuss in the next section have the property that any of their leading principal submatrices are in the same class (of lower order). Therefore, the discussion can be restricted to

$$
p(A):=\frac{\operatorname{det} A}{\operatorname{det} A^{\prime}} .
$$

Proposition 3.1. The partial derivative of $p(A)=\operatorname{det} A / \operatorname{det}\left(A^{\prime}\right)$ w.r.t. the entry $a_{i j}$ is given by

$$
\frac{\partial p(A)}{\partial a_{i j}}=(-1)^{i+j} \frac{\operatorname{det} A_{n j} \operatorname{det} A_{i n}}{\left(\operatorname{det} A^{\prime}\right)^{2}}, \quad i, j=1, \ldots, n .
$$

Proof. By Laplacian expansion along row $i$, we obtain

$$
\frac{\partial p(A)}{\partial a_{i j}}=\left\{\begin{array}{l}
(-1)^{i+j} \frac{\operatorname{det} A_{i j} \operatorname{det} A^{\prime}-\operatorname{det} A_{i j}^{\prime} \operatorname{det} A}{\left(\operatorname{det} A^{\prime}\right)^{2}} \text { if } i, j<n, \\
(-1)^{i+j} \frac{\operatorname{det} A_{i j}}{\operatorname{det} A^{\prime}} \quad \text { if } i=n \text { or } j=n .
\end{array}\right.
$$

To show (3.5) for $i, j<n$ we make use of a special case of Sylvester's determinantal identity (e.g., [12, p. 22]): Let $C \in \mathbf{R}^{n \times n}, \alpha \subseteq\{1,2, \ldots, n\}$ be a fixed set of cardinality $n-2$, and let $\{1,2, \ldots, n\} \backslash \alpha=\{i, j\}$ with $i<j$. Define the 2-by-2 matrix $B$ by

$$
\begin{aligned}
& b_{11}:=\operatorname{det} C[\alpha \cup\{i\}], \\
& b_{12}:=\operatorname{det} C[\alpha \cup\{i\}, \alpha \cup\{j\}], \\
& b_{21}:=\operatorname{det} C[\alpha \cup\{j\}, \alpha \cup\{i\}], \\
& b_{22}:=\operatorname{det} C[\alpha \cup\{j\}] .
\end{aligned}
$$

Then it holds that

$$
\operatorname{det} B=\operatorname{det} C[\alpha] \operatorname{det} C \text {. }
$$

Now, we interchange in $A$ rows 1 and $i$ and columns 1 and $j$ and apply (3.7) to the resulting matrix denoted by $C$ with $\alpha=\{2,3, \ldots, n-1\}$. Since

$$
\begin{aligned}
\operatorname{det} C[\alpha] & =(-1)^{i+j} \operatorname{det} A_{i j}^{\prime}, \\
\operatorname{det} C[\{1, \ldots, n-1\}] & =\operatorname{det} A^{\prime}, \\
\operatorname{det} C[\{2, \ldots, n\}] & =(-1)^{i+j} \operatorname{det} A_{i j}, \\
\operatorname{det} C[\{1, \ldots, n-1\} \mid\{2, \ldots, n\}] & =(-1)^{j-1} \operatorname{det} A_{n j}, \\
\operatorname{det} C[\{2, \ldots, n\} \mid\{1, \ldots, n-1\}] & =(-1)^{i-1} \operatorname{det} A_{i n},
\end{aligned}
$$


formula (3.7) yields

$$
\operatorname{det} A_{i j}^{\prime} \operatorname{det} A=\operatorname{det} A_{i j} \operatorname{det} A^{\prime}-\operatorname{det} A_{n j} \operatorname{det} A_{i n} .
$$

Combining with (3.6), we obtain (3.5) for $i, j<n$.

Note that $\operatorname{det} A_{n j}$ and $\operatorname{det} A_{i n}$ appear in the cofactor form of the inverse of $A$. Therefore, we consider in the following section sets of matrices with identically signed inverses.

\section{Applications.}

4.1. Inverse nonnegative matrices. We will make use of the following property of the inverse nonnegative matrices.

Proposition 4.1 (see [16]). Let $[A]=[\underline{A}, \bar{A}]$ be a matrix interval and $\underline{A}$ and $\bar{A}$ be inverse nonnegative. Then $[A]$ is inverse nonnegative and $\bar{A}^{-1} \leq \underline{A}^{-1}$.

For results on properties of inverse nonnegative matrix intervals the reader is referred to [23] and for the construction of $[A]^{H}[b]$ in this case to [21] and the references therein. We will focus here on the applicability of the interval Gaussian elimination. It is known that interval Gaussian elimination may break down for inverse nonnegative interval matrices; see, e.g., [20, p. 160]. We apply the pivot tightening approach from section 3 .

We assume now that each $A \in[A]$ has the property that all its leading principal submatrices are inverse nonnegative. By $[19$, p. 24$]$ this is equivalent to the property that $A$ allows a factorization $A=L D U$, where $L$ and $U$ are inverse nonnegative lower and upper triangular matrices, respectively, whose diagonal entries are all one, and $D$ is a diagonal matrix with positive diagonal entries. According to Proposition 4.1 the condition on $[A]=[\underline{A}, \bar{A}]$ is fulfilled if all leading principal submatrices of $\underline{A}$ and $\bar{A}$ are inverse nonnegative.

THEOREM 4.2. If all leading principal submatrices of $[A]=[\underline{A}, \bar{A}]$ are inverse nonnegative, then the range of $p(A)$ over $[A]$ is given by

$$
p([A])=[p(\underline{A}), p(\bar{A})] .
$$

Proof. For $\underline{A} \leq A \leq \bar{A}$ it follows from Proposition 4.1 that

$$
\left(\bar{A}^{-1}\right)_{n n} \leq\left(A^{-1}\right)_{n n} \leq\left(\underline{A}^{-1}\right)_{n n} .
$$

Formula (4.1) is now a consequence of $\left(A^{-1}\right)_{n n}=\frac{1}{p(A)}$.

The practical application of (4.1) requires running in parallel to the interval Gaussian elimination two instances of ordinary Gaussian elimination applied to $\underline{A}$ and $\bar{A}$. In the $k$ th step, both pivots span the interval given on the right-hand side of (4.1).

4.2. Totally nonnegative matrices. A matrix $A \in \mathbf{R}^{n \times n}$ is called totally nonnegative or totally positive if all its minors are nonnegative or positive, respectively. These matrices appear in mechanics and in many branches of mathematics. If $A$ is nonsingular and totally nonnegative, then so too is each leading principal submatrix and $A$ is a $P$-matrix. For further properties of these matrices the reader is referred to $[3]$.

A suitable partial order for the totally nonnegative matrices is the checkerboard order. For $A, B \in \mathbf{R}^{n \times n}$ define

$$
A \leq^{*} B:=(-1)^{i+j} a_{i j} \leq(-1)^{i+j} b_{i j}, \quad i, j=1,2, \ldots, n .
$$


This partial order is related to the usual entrywise partial order by

$$
A \leq^{*} B \Leftrightarrow A^{*} \leq B^{*}, \text { where } A^{*}:=S A S, S:=\operatorname{diag}\left(1,-1, \ldots,(-1)^{n+1}\right),
$$

is the checkerboard transformation.

A matrix interval $[\underline{A}, \bar{A}]$ w.r.t. the usual entrywise partial order can be represented as an interval $[\downarrow A, \uparrow A]^{*}$ w.r.t. the checkerboard order, where

$$
\begin{aligned}
& (\downarrow A)_{i j}:= \begin{cases}\underline{a}_{i j} & \text { if } i+j \text { is even, } \\
\bar{a}_{i j} & \text { if } i+j \text { is odd, }\end{cases} \\
& (\uparrow A)_{i j}:= \begin{cases}\bar{a}_{i j} & \text { if } i+j \text { is even, } \\
\underline{a}_{i j} & \text { if } i+j \text { is odd. }\end{cases}
\end{aligned}
$$

It is conjectured in $[7]^{2}$ that if $\downarrow A$ and $\uparrow A$ are nonsingular and totally nonnegative, then the whole matrix interval $[\downarrow A, \uparrow A]^{*}$ is nonsingular and totally nonnegative. This conjecture is settled for the totally positive and nonsingular tridiagonal totally nonnegative matrices [7], almost totally positive matrices [10], and interval matrices $[A]$ for which the index sum $i+j$ of all degenerate entries $\left[a_{i j}\right]$ has the same parity [7]. In [9] a subset of cardinality of at most $2^{2 n-1}$ vertex matrices is given, from which the total nonnegativity of the entire matrix interval can be inferred.

Note that if $A$ is nonsingular and totally nonnegative, then $0 \leq^{*} A^{-1}$ and, therefore, $0 \leq\left(A^{-1}\right)^{*}=\left(A^{*}\right)^{-1}$. Since $A^{*}$ is inverse nonnegative, all results for inverse nonnegative matrices carry over to the totally nonnegative matrices by the checkerboard transformation; e.g., if $A$ and $B$ are nonsingular and totally nonnegative, then it follows that $A \leq^{*} B \Rightarrow B^{-1} \leq^{*} A^{-1}$. For results on the calculation of $[A]^{H}[b]$ under special sign conditions posed on [b], cf. [6].

In [6] it was shown that the interval Gaussian elimination may fail if it is applied to a nonsingular totally nonnegative interval matrix. As the most important application of the approach presented in [18], the pivot tightening (3.3) was employed for these interval matrices using the fact that the range of the determinant on such an interval matrix is given by

$$
\operatorname{det} A\left([\downarrow A, \uparrow A]^{*}\right)=[\operatorname{det}(\downarrow A), \operatorname{det}(\uparrow A)] .
$$

However, inspection of (3.5) or the use of the results in subsection 4.1 and application of the checkerboard transformation shows that even the exact range of the pivot of ordinary Gaussian elimination can be given.

Corollary 4.3. If $[A]=[\downarrow A, \uparrow A]^{*}$ is nonsingular and totally nonnegative, then the range of $p(A)$ over $[A]$ is given by

$$
p\left([\downarrow A, \uparrow A]^{*}\right)=[p(\downarrow A), p(\uparrow A)] .
$$

Example 4.4. We consider the example in [6], also treated in [18]. Let

$$
[A]:=\left(\begin{array}{ccc}
{[4,5]} & {[2,3]} & 1 \\
{[2,3]} & 4 & {[2,3]} \\
1 & {[2,3]} & {[4,5]}
\end{array}\right) .
$$

\footnotetext{
${ }^{2}$ Note that Theorem 1 in [6] is not correct.
} 
Taking (4.3) into account, it is easily checked that $[A]$ is nonsingular and totally nonnegative. Interval Gaussian elimination results in the interval

$$
\left[a_{33}\right]^{(3)}=\left[-\frac{79}{700}, \frac{5519}{1280}\right]
$$

which contains zero, and breaks down. ${ }^{3}$ Using (4.3) the pivot tightening approach from [18] gives for this entry $\left[a_{33}\right]^{(3)} \cap \frac{[6,64]}{[7,16]}=\left[\frac{3}{8}, \frac{5519}{1280}\right]$, whereas (4.4) results in the smaller interval $\left[\frac{6}{7}, 4\right]$.

4.3. Inverse $M$-matrices. A matrix $A \in \mathbf{R}^{n \times n}$ is called an inverse $M$-matrix if it is the inverse of an $M$-matrix. Inverse $M$-matrices are entrywise nonnegative and $P$-matrices; each leading principal submatrix is likewise an inverse $M$-matrix. For further properties of these matrices the reader is referred to [13]. Sufficient conditions for a real matrix to be an inverse $M$-matrix and applications of these matrices can be found in [25].

We will make use of the following proposition.

Proposition 4.5 (see [15]). A matrix interval is an inverse $M$-matrix interval if and only if all its vertex matrices are inverse $M$-matrices.

Example 4.7 below shows that interval Gaussian elimination may fail if it is applied to such a matrix interval. Pivot tightening here is more involved than in (4.1) and (4.4).

THEOREM 4.6. If $[A] \in \mathbf{I R}^{n \times n}$ is an inverse $M$-matrix interval, then the range of $p(A)$ over $[A]$ is given by

$$
\begin{aligned}
& ([A])=\left[p\left(A^{l}\right), p\left(A^{u}\right)\right], \quad \text { where } \\
A^{l}:= & \left(\begin{array}{cccc}
\underline{a}_{11} & \cdots & \underline{a}_{1, n-1} & \bar{a}_{1 n} \\
\vdots & & \vdots & \vdots \\
\underline{a}_{n-1,1} & \cdots & \underline{a}_{n-1, n-1} & \bar{a}_{n-1, n} \\
\bar{a}_{n 1} & \cdots & \bar{a}_{n, n-1} & \underline{a}_{n n}
\end{array}\right), \\
A^{u}:= & \left(\begin{array}{cccc}
\bar{a}_{11} & \cdots & \bar{a}_{1, n-1} & \underline{a}_{1 n} \\
\vdots & & \vdots & \vdots \\
\bar{a}_{n-1,1} & \cdots & \bar{a}_{n-1, n-1} & \underline{a}_{n-1, n} \\
\underline{a}_{n 1} & \cdots & \underline{a}_{n, n-1} & \bar{a}_{n n}
\end{array}\right) .
\end{aligned}
$$

Proof. According to the sign condition that the inverse of an inverse $M$-matrix $A$ must have positive diagonal entries and all of its off-diagonal entries must be nonpositive, we obtain

$$
\begin{aligned}
& 0<\operatorname{det} A_{i i}, \quad i=1, \ldots, n, \\
& \operatorname{sgn}\left(\operatorname{det} A_{i j}\right) \in\left\{0,(-1)^{i+j+1}\right\}, \quad i, j=1, \ldots, n, i \neq j .
\end{aligned}
$$

Consider in Proposition 3.1 first the case $i=n$ or $j=n$. It follows that the partial derivative of $p$ w.r.t. the entries in the last row or column is nonpositive (note that

\footnotetext{
${ }^{3}$ It should be noted that the interval Gaussian elimination would not fail, were $\left[a_{22}\right]$ chosen as the first pivot. The same applies to Example 4.7.
} 
$\left.0<\operatorname{det} A^{\prime}\right)$ with the exception of $a_{n n}$ for which the partial derivative is positive. In the case $i, j<n$, note that $\operatorname{sgn}\left(\operatorname{det} A_{n j} \operatorname{det} A_{i n}\right) \in\left\{0,(-1)^{i+j}\right\}$. Therefore, the partial derivative of $p$ w.r.t. an entry of $A^{\prime}$ is always nonnegative.

Remark 4.6.1. An alternative proof in the case $i, j<n$ is as follows: ${ }^{4}$ By permutation similarity of the inverse $M$-matrices it suffices to consider only entry $a_{12}$. The inequality

$$
\operatorname{det} A_{12} \operatorname{det} A^{\prime} \leq \operatorname{det} A_{12}^{\prime} \operatorname{det} A
$$

follows from the inequality [14, Theorem 2.1(ii)]

$$
(A[\alpha])^{-1} \leq A^{-1}[\alpha]
$$

which is valid for inverse $M$-matrices by choosing $\alpha=\{1, \ldots, n-1\}$.

Example 4.7. Let

$$
[A]:=\left(\begin{array}{ccc}
{[1,4]} & {\left[\frac{1}{2}, \frac{\sqrt{2}}{2}\right]} & \frac{\sqrt{2}}{2} \\
\frac{\sqrt{2}}{2} & 1 & {\left[\frac{1}{2}, \frac{\sqrt{2}}{2}\right]} \\
\frac{1}{2} & \frac{\sqrt{2}}{2} & 1
\end{array}\right) .
$$

It is easily checked that all eight vertex matrices are inverse $M$-matrices, and by Proposition 4.5 it follows that $[A]$ is an inverse $M$-matrix interval. Interval Gaussian elimination results in the interval

$$
\left[a_{33}^{(3)}\right]=\left[-\frac{4 \sqrt{2}+1}{64}, 1-\frac{\sqrt{2}}{16}\right],
$$

which contains zero, and breaks down. By Theorem 4.6 this pivot can be tightened to $\left[\frac{1}{2}, 1-\frac{\sqrt{2}}{4}\right]=[0.5,0.6464 \ldots$.$] .$

If pivot tightening is applied in all steps, the computation of $p\left(A^{l}\right)$ (and similarly of $p\left(A^{u}\right)$ ) requires running in parallel to the interval Gaussian elimination an extension of ordinary Gaussian elimination. The elimination algorithm is applied to the submatrices of $[A]$ in the order indicated in Figure 4.1. The part which is recomputed or newly computed is inside the shaded area.

5. A related determinantal function. In a similar way we can determine monotonicity of the related determinantal function

$$
d(A):=\operatorname{det} A \operatorname{det} A^{\prime}
$$

which appears, e.g., in the matricial description of the Cholesky decomposition [5, p. 38].

TheOrem 5.1. Let $[A] \in \mathbf{I R}^{n \times n}$.

(i) If $[A]$ fulfills the assumption of Theorem 4.2, then the range of $d(A)$ over $[A]$ is given by

$$
d([A])=[d(\underline{A}), d(\bar{A})]
$$

\footnotetext{
${ }^{4}$ This was pointed out to us by Professor Charles R. Johnson.
} 


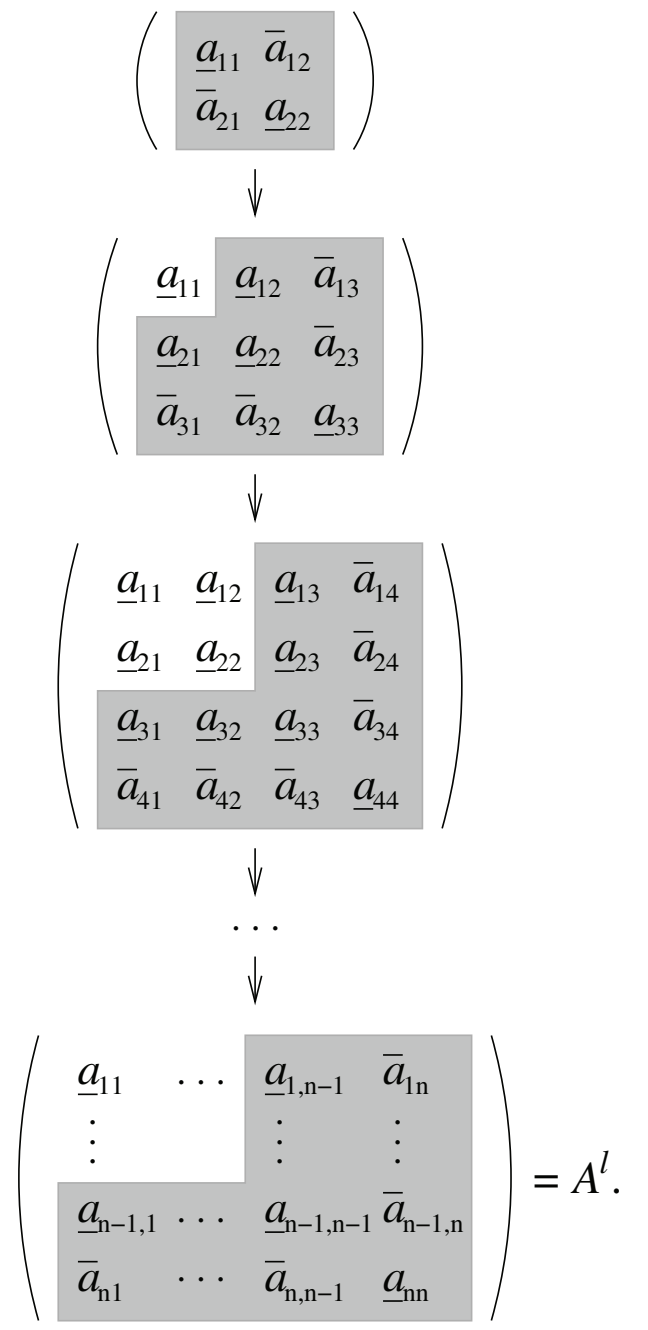

FIG. 4.1. Elimination algorithm applied to submatrices of $[A]$.

(ii) If $[A]$ is an inverse $M$-matrix interval, it holds that

$$
d([A])=\left[d\left(A^{1}\right), d\left(A^{2}\right)\right]
$$

where

$$
A^{1}:=\left(\begin{array}{cccc}
\underline{a}_{11} & \bar{a}_{12} & \ldots & \bar{a}_{1 n} \\
\bar{a}_{21} & \underline{a}_{22} & \ddots & \vdots \\
\vdots & \ddots & \ddots & \bar{a}_{n-1, n} \\
\bar{a}_{n 1} & \cdots & \bar{a}_{n, n-1} & \underline{a}_{n n}
\end{array}\right)
$$




$$
A^{2}:=\left(\begin{array}{cccc}
\bar{a}_{11} & \underline{a}_{12} & \cdots & \underline{a}_{1 n} \\
\underline{a}_{21} & \bar{a}_{22} & \ddots & \vdots \\
\vdots & \ddots & \ddots & \underline{a}_{n-1, n} \\
\underline{a}_{n 1} & \cdots & \underline{a}_{n, n-1} & \bar{a}_{n n}
\end{array}\right) .
$$

Proof. Similarly as in (3.6) we obtain

$$
\frac{\partial d(A)}{\partial a_{i j}}= \begin{cases}(-1)^{i+j}\left(\operatorname{det} A_{i j} \operatorname{det} A^{\prime}+\operatorname{det} A_{i j}^{\prime} \operatorname{det} A\right) & \text { if } i, j<n, \\ (-1)^{i+j} \operatorname{det} A_{i j} \operatorname{det} A^{\prime} & \text { if } i=n \text { or } j=n .\end{cases}
$$

Let $[A]$ first fulfill the assumption of Theorem 4.2. From the sign condition for the inverse $B$ of an inverse nonnegative matrix $A, B=A^{-1}$, we obtain

$$
0 \leq b_{i j}=(-1)^{i+j} \frac{\operatorname{det} A_{j i}}{\operatorname{det} A}
$$

in particular for $i=j=n$,

$$
0<b_{n n}=\frac{\operatorname{det} A^{\prime}}{\operatorname{det} A}
$$

whence

$$
\operatorname{sgn}(\operatorname{det} A)=\operatorname{sgn}\left(\operatorname{det} A^{\prime}\right) .
$$

If $i, j<n$ and $B^{\prime}:=\left(A^{\prime}\right)^{-1}$, it follows that

$$
\frac{\partial d(A)}{\partial a_{i j}}=\left(b_{j i}+b_{j i}^{\prime}\right) \operatorname{det} A^{\prime} \operatorname{det} A \geqq 0 .
$$

If $i=n$ or $j=n$, the proof of the nonnegativity of the partial derivative of $d$ w.r.t. $a_{i j}$ is similar. Therefore, $d$ is monotone increasing. This proves (i).

If $[A]$ is an inverse $M$-matrix interval, we obtain from the sign condition (4.5) that $d$ is monotone increasing w.r.t. the diagonal entries and monotone decreasing w.r.t. the off-diagonal entries. This proves (ii).

6. Methods for structured systems. The results for interval Gaussian elimination apply to some algorithms for the solution of structured systems of linear interval equations. Interval variants for the respective ordinary methods are obtained by replacing the real numbers by the related intervals and the real operations by the respective interval operations.

If $[A]$ is symmetric, i.e., $[A]=[A]^{T}$, then the symmetric solution set

$$
\Sigma_{\text {Sym }}([A],[b]):=\left\{x \in \mathbf{R}^{n} \mid A x=b, A=A^{T}, A \in[A], b \in[b]\right\}
$$

is considered. The interval Cholesky method [2] can be used to enclose this set. Each step requires the division by an interval which may contain zero even if all symmetric matrices $A \in[A]$ are positive definite; an example is provided by Example 4.4. For each step of the ordinary Cholesky method, the divisor can be represented as the square root of the quotient of two succeeding leading principal minors as in (3.4) (cf. [5, p. 38]), so that the result for the interval Gaussian elimination applies. 
Let $[A]$ be a Toeplitz interval matrix (of order $n+1$ ), i.e., there are intervals $\left[a_{i}\right]$, $i=-n, \ldots, n$, such that $\left[a_{i j}\right]=\left[a_{j-i}\right], i, j=1, \ldots, n+1$, and define by $[A]_{T}$ the set of all real Toeplitz matrices contained in $[A]$. Then one restricts the solution set (1.1) to the Toeplitz solution set

$$
\Sigma_{T}([A],[b]):=\left\{x \in \mathbf{R}^{n} \mid A x=b, A \in[A]_{T}, b \in[b]\right\} .
$$

Interval variants of the elimination procedure of Bareiss [4] and of the recurrence relations for the inverse of a Toeplitz matrix [24], [26] are investigated in [8]. Even if all matrices $A \in[A]_{T}$ have only nonzero leading principal minors, these interval algorithms may break down due to division by an interval containing zero. An example is the interval matrix in [22]. For the ordinary (real) version of both algorithms the divisor can be represented as quotient of two successive leading principal minors (cf. Corollary 1 in [4] and [26, p. 276]), so that for both algorithms the results for the interval Gaussian elimination also apply.

If pivot tightening is used in the symmetric case, then for the three classes of matrix intervals considered in section 4 , the respective vertex matrices are symmetric. In the Toeplitz case, the two vertex matrices are Toeplitz matrices in the case of an inverse nonnegative or nonsingular totally nonnegative matrix interval, but in general not in the case of an inverse $M$-matrix interval.

An elimination process very well suited for totally nonnegative matrices is Neville elimination [11]. Here, zeros in a column below the main diagonal of an $n$-by- $n$ matrix are produced by adding to each row an appropriate multiple of the previous one (instead of using a fixed row with a fixed pivot as in Gaussian elimination). Lemma 2.6 in [11] shows that all the pivots of Neville elimination are nonzero if and only if the column-initial minors $\operatorname{det} A[\alpha \mid\{1, \ldots, k\}]$, where $\alpha$ is a subset of $k$ successive elements of $\{1, \ldots, n\}$, are nonzero, $k=1, \ldots, n$. Moreover, in this case, Neville elimination can be carried out without row interchanges. This suggests that the column-initial minors play a role in Neville elimination similar to that of the leading principal minors in Gaussian elimination. Formula (2.8) in [11] shows that the pivots in the $k$ th step of Neville elimination are just the quotients of a columninitial minor of order $k$ and its leading principal minor which is column-initial of order $k-1$. So we can apply Proposition 3.1 and the results from subsection 4.2.

7. Conclusions. We have shown how for some classes of interval matrices the interval pivots in the performance of the interval Gaussian elimination can be tightened such that the shrunken interval does not contain zero, thereby avoiding a breakdown of the algorithm. As a positive side effect, the tightening often leads to a smaller enclosure of the solution set, so that the approach is recommended not only merely for avoiding a breakdown. The extra computational effort consists of two or four instances of ordinary Gaussian elimination, depending on the class of matrices under consideration. However, to obtain verified results, these parallel runs of Gaussian elimination should be performed in interval arithmetic, too. The approach easily extends to some algorithms for solving structured systems of linear interval equations and may be applied to other classes of matrices with identically signed inverses.

Acknowledgments. We thank Professor Charles R. Johnson for his comments on the case of inverse $M$-matrices and Andrew P. Smith for his careful reading of the manuscript. 
[1] G. Alefeld and J. Herzberger, Introduction to Interval Computations, Academic Press, New York, 1983.

[2] G. Alefeld and G. Mayer, The Cholesky method for interval data, Linear Algebra Appl., 194 (1993), pp. 161-182.

[3] T. Ando, Totally positive matrices, Linear Algebra Appl., 90 (1987), pp. 165-219.

[4] E. H. BAREISS, Numerical solution of linear equations with Toeplitz and vector Toeplitz matrices, Numer. Math., 13 (1969), pp. 404-424.

[5] F. R. Gantmacher, The Theory of Matrices, Vol. 1, Chelsea Publishing, New York, 1977.

[6] J. GarlofF, Totally nonnegative interval matrices, in Interval Mathematics 1980, K. Nickel, ed., Academic Press, New York, London, Toronto, 1980, pp. 317-327.

[7] J. GarlofF, Criteria for sign regularity of sets of matrices, Linear Algebra Appl., 44 (1982), pp. $153-160$.

[8] J. GARLOFF, Solution of linear equations having a Toeplitz interval matrix as coefficient matrix, Opuscula Math., 2 (1986), pp. 33-45.

[9] J. GARLOFF, Vertex implications for totally nonnegative matrices, in Total Positivity and Its Applications, M. Gasca and C. A. Micchelli, eds., Kluwer Academic Publishers, Dordrecht, Boston, London, 1996, pp. 103-107.

[10] J. Garloff, Intervals of almost totally positive matrices, Linear Algebra Appl., 363 (2003), pp. 103-108.

[11] M. Gasca And J. M. Peña, Total positivity and Neville elimination, Linear Algebra Appl., 165 (1992), pp. $25-44$.

[12] R. A. Horn And C. R. Johnson, Matrix Analysis, Cambridge University Press, Cambridge, UK, 1994.

[13] C. R. Johnson, Inverse M-matrices, Linear Algebra Appl., 47 (1982), pp. 195-216.

[14] C. R. Johnson And R. L. Smith, Almost principal minors of inverse M-matrices, Linear Algebra Appl., 337 (2001), pp. 253-265.

[15] C. R. Johnson And R. L. Smith, Intervals of inverse $M$-matrices, Reliab. Comput., 8 (2002), pp. 239-243.

[16] J. KuTTLER, A fourth-order finite-difference approximation for the fixed membrane eigenproblem, Math. Comp., 25 (1971), pp. 237-256.

[17] G. Mayer, A contribution to the feasibility of the interval Gaussian algorithm, Reliab. Comput., 12 (2006), pp. 79-98.

[18] J. MAYER, An approach to overcome division by zero in the interval Gauss algorithm, Reliab. Comput., 8 (2002), pp. 229-237.

[19] K. Metelmann, Inverspositive Bandmatrizen und totalnichtnegative Green'sche Matrizen, Dissertation, University of Cologne, Cologne, Germany, 1972.

[20] A. Neumaier, Interval Methods for Systems of Equations, Cambridge University Press, Cambridge, UK, 1990.

[21] S. Ning And R. B. Kearfott, A comparison of some methods for solving linear interval equations, SIAM J. Numer. Anal., 34 (1997), pp. 1289-1305.

[22] K. Reichmann, Abbruch beim Intervall-Gauss-Algorithmus, Computing, 22 (1979), pp. 355361.

[23] J. Rohn, Inverse-positive interval matrices, Z. Angew. Math. Mech., 67 (1987), pp. T492-T493.

[24] W. F. Trench, An algorithm for the inversion of finite Toeplitz matrices, J. Soc. Indust. Appl. Math., 12 (1964), pp. 515-522.

[25] R. A. Willoughby, The inverse M-matrix problem, Linear Algebra Appl., 18 (1977), pp. 7594.

[26] S. ZoHAR, The solution of a Toeplitz set of linear equations, J. ACM, 21 (1974), pp. 272-276. 